\title{
The Impact of Fructo-Oligosaccharides on Gut Permeability and Inflammatory Responses in the Cecal Mucosa Quite Differs between Rats Fed Semi-Purified and Non-Purified Diets
}

\author{
Tomomi GendA ${ }^{1}$, Takashi Kondo ${ }^{1}$, Shingo Hino ${ }^{2}$, Shunsaku SugiurA ${ }^{3}$, \\ Naomichi NisHIMURA ${ }^{2}$ and Tatsuya MORITA ${ }^{2, *}$ \\ ${ }^{1}$ Department of Bioscience, Graduate School of Science and Technology, Shizuoka University, \\ Shizuoka 422-8529, Japan \\ ${ }^{2}$ College of Agriculture, Academic Institute, Shizuoka University, Shizuoka 422-8529, Japan \\ ${ }^{3}$ Department of Agriculture, Graduate School of Integrated Science and Technology, \\ Shizuoka University, Shizuoka 422-8529, Japan
}

(Received March 17, 2018)

\begin{abstract}
Summary The effects of fructo-oligosaccharides (FOS) on gut-barrier function are still controversial in human and animal studies. Diet conditions would be a major factor for the controversy in animal studies. We fed rats a semi-purified (SP) or a non-purified diet (NP) with or without FOS $(60 \mathrm{~g} / \mathrm{kg}$ diet) for 9 (experiment 1) or $10 \mathrm{~d}$ (experiment 2). We assessed microbial fermentation, gut permeability, and inflammatory responses in the cecum (experiment 1), and mucus layer in the cecum, intestinal transit time and microbiota composition (experiment 2). FOS supplementation induced a very acidic fermentation due to the accumulation of lactate and succinate in SP, while short-chain fatty acids were major products in NP. Gut permeability estimated by urinary chromium-EDTA excretion, bacterial translocation into mesenteric lymph nodes, myeloperoxidase activity, and expressions of the inflammatory cytokine genes in the cecal mucosa were greater in SP+FOS than in SP, but these alterations were not observed between NP and NP+FOS (experiment 1). FOS supplementation destroyed the mucus layer on the epithelial surface in SP, but not in NP. Intestinal transit time was 3-fold longer in SP+FOS than in SP, but this was not the case between NP and $\mathrm{NP}+\mathrm{FOS}$. Lower species richness of cecal microbiota was manifest solely in SP+FOS (experiment 2). These factors suggest that impact of FOS on gut permeability and inflammatory responses in the cecal mucosa quite differs between SP and NP. Increased gut permeability in SP+FOS could be evoked by the disruption of the mucus layer due to stasis of the very acidic luminal contents.
\end{abstract}

Key Words fructo-oligosaccharides, gut permeability, semi-purified diet, non-purified diet, rats

There has been a long-lasting debate over the effects of fructo-oligosaccharides (FOS) and short-chain fructans on gut permeability in animals and humans (1, 2). In animal studies, a series of publications by Ten Bruggencate et al. $(3,4$,$) and others (5,6)$ indicated that short-term ingestion (generally up to $2 \mathrm{wk}$ ) of FOS, galacto-oligosaccharides or rapidly fermentable carbohydrates increased gut permeability in rats and mice, as evidenced by the increases in the urinary $\mathrm{Cr}$ EDTA excretion, commensal bacteria translocation to the organs, and salmonella invasion. In contrast, several publications have insisted that consumption of FOS and short-chain fructans could reinforce the mucosal barrier function in the large intestine, because the consumption of FOS and short-chain fructans favorably altered the intestinal mucosal architecture including the increases in crypt depth, goblet cell numbers, mucus layer thickness on the epithelial surface, and the trans-

\footnotetext{
*To whom correspondence should be addressed.
}

E-mail:morita.tatsuya@shizuoka.ac.jp mucosal resistance in the cecum $(7-10)$. These contradictory findings were obtained in animals fed 5 to $10 \%$ supplemental FOS in semi-purified (SP) or non-purified diet (NP) for different ingestion periods.

Many factors could be involved in the above-mentioned different findings of FOS on gut permeability. Our recent study, however, showed that FOS-induced increase in the gut permeability in rats fed a SP was a transient phenomenon before adaptation of the gut bacteria to FOS (11). Cecal fermentation in rats fed 6\% FOS diet for $1 \mathrm{wk}$ was characterized by a very low cecal $\mathrm{pH}$ (well below 6.0) accompanying the accumulation of lactate and succinate, which are stronger acids than short-chain fatty acids (SCFA) but are absorbed more slowly (12). In such a cecal environment, we found that the mucus layer on the epithelial surface in the cecum was completely destroyed, and this might be associated with the increased gut permeability (11). But in rats fed the same diet for $8 \mathrm{wk}$, at which time the cecal $\mathrm{pH}$ became almost neutral and SCFA were major fermentation products, the gut permeability returned to its nor- 
mal level (11). Thus, the most obvious consideration for the increased gut permeability in the short-term ingestion of FOS should be "dysfermentation" (abnormal fermentation) and possibly dysbiosis as well, presumably due to an excessive supply of a rapidly fermentable carbohydrate.

In contrast to SP, which is made of highly digestible refined ingredients, the primary ingredients of NP are provided from natural sources, such as corn, wheat bran, defatted soybean, defatted rice bran, fishmeal, defatted milk, soybean oil, and brewer's yeast. Thus, NP contains many types of non-digestible ingredients. Previous studies showed that digestion-resistant proteins substantially modified fermentation pattern of rapidly fermentable carbohydrates such as high-amylose cornstarch and FOS to shift the production toward more SCFA and less succinate and lactate through a correction of imbalance of the carbohydrate/nitrogen supply ratio as fermentation substrates $(13,14)$. In addition, some poorly fermentable bulky fibers in NP may shorten large bowel transit time of the luminal contents and protect FOS from full fermentation in the cecum (15). Collectively, we speculate that supplemental FOS in NP may elicit different features of the cecal fermentation and thereby different outcomes on the gut permeability, and immunological and microbial alterations in rats. Most of the prebiotic effects on the large bowel functions are indirectly exerted through a shift in composition of the intestinal flora and the enhanced production of organic acids and perhaps other bacterial products (16). In this context, it would be of value to clarify which physiologic effects by FOS are exerted under which diet conditions such as SP and NP.

In this study, therefore, we aimed to determine whether feeding a NP compared with a SP supplemented with or without FOS differently impacts the gut permeability in rats, judging from urinary Cr-EDTA excretion, bacterial translocation to mesenteric lymph nodes (MLN), myeloperoxidase activity (MPO) and expression of inflammatory cytokine genes in the cecal mucosa and the mucus layer on the epithelial surface in the cecum. In addition, to get more insight into the causative factors that affect differences in the cecal fermentation of FOS and the related consequences between rats fed SP and NP, we compared the gastrointestinal transit time of the luminal contents and the microbial composition in rats fed SP or NP with or without supplemental FOS.

\section{MATERIALS AND METHODS}

Materials. FOS, being composed of 44\% 1-kestose, $46 \%$ nystose, and $10 \% 1$-f- $\beta$-fructofuranosyl nystose, was purchased from Meiji Seika (Meioligo ${ }^{\circledR}$ P, Tokyo, Japan). A NP was purchased from Oriental Yeast Co., Ltd. $\left(\mathrm{MF}^{\circledR}\right.$, Tokyo, Japan). According to the manufacturer's information, composition of the NP diet is shown in Table 1 and Supplemental Online Material, Table S1. According to the method of dietary fiber measurement by Prosky (18), the NP contained 17\% total dietary fiber and $11 \%$ protease-resistant protein.

Care of animals. All aspects of animal care were
Table 1. Composition of semi-purified and non-purified diets.

\begin{tabular}{lc}
\hline & $\mathrm{g} / \mathrm{kg}$ diet \\
\hline Semi-purified diet & \\
Casein & 250 \\
Cornstarch $_{\text {Corn oil }}$ & 652.5 \\
Vitamin mixture $^{1}$ & 50 \\
Mineral mixture $^{1}$ & 10 \\
Choline bitartrate $^{2}$ & 35 \\
Non-purified diet & 2.5 \\
Crude protein $^{2}$ & \\
Non-nitrogenous matter & 250.8 \\
Crude fat $_{\text {Ash }}$ & 600.4 \\
Crude fiber $^{2}$ & 55.4 \\
& 63.0 \\
& 30.4
\end{tabular}

\footnotetext{
${ }^{1}$ According to AIN-76 (17).

${ }^{2}$ Non-purified powder diet (MF) was purchased from Oriental Yeast Co., Ltd.

Nutrient content is from http://www.oyc-bio.jp/pages/ animal_products/feed/ingredient.

Further details of chemically defined amounts of each vitamin and mineral are shown in Supplemental Table 1. As for further information, the non-purified diet contained $17 \%$ total dietary fiber according to the method of Prosky (18).
}

under the oversight of the Institutional Animal Care and Use Committee of Shizuoka University under accepted guidelines (approval no. 26-14, 27-14). Male Wistar rats ( $6 \mathrm{wk}$ old) were purchased from Shizuoka Laboratory Animal Center (Shizuoka, Japan). The rats were housed individually in screen-bottomed stainless-steel cages in a temperature $\left(23 \pm 1^{\circ} \mathrm{C}\right)$ and light (lights on from $07: 00$ to $19: 00)$ controlled room. The rats were acclimatized for $7 \mathrm{~d}$ and fed a SP (Table 1). The rats were subsequently allocated to groups based on body weight in order to normalize body weights across the groups.

Animal experiments. Two animal experiments were separately conducted to provide enough data for cecal variables (experiment 1, epithelial mucus layer; experiment 2, epithelial mucus layer in the cecum) and $\mathrm{Cr}$ EDTA excretion (experiment 1, urinary excretion; experiment 2, fecal excretion).

Experiment 1. Twenty-four rats, weighing 130$150 \mathrm{~g}$, were allocated to 4 groups of 6 rats each. Rats were allowed free access to water and either SP or NP with or without supplemental FOS ( $60 \mathrm{~g} / \mathrm{kg}$ diet) for $9 \mathrm{~d}$. Supplementation of FOS was performed by replacement of an equal amount of cornstarch in the SP or an equal amount of the NP as a whole. There were thus 4 dietary groups, i.e., SP, SP+FOS, NP and NP+FOS. On day 7, rats were moved to metabolic cages where they received an oral administration of Cr-EDTA (50 mg per rat) at $1300 \mathrm{~h}$; urine was then collected for $48 \mathrm{~h}$. The rats were killed by decapitation under pentobarbital anesthesia at $1300 \mathrm{~h}$ on day 9. Five samples of MLNs, to which the lymph from the cecum and the colon drain (11), were collected under aseptic conditions and used for analy- 
Table 2. Food intake, body weight gain, and cecal variables in male Wistar rats fed either SP and NP with or without $60 \mathrm{~g}$ of FOS/ kg diet for $9 \mathrm{~d}$ (experiment 1).

\begin{tabular}{|c|c|c|c|c|c|c|c|}
\hline & \multirow{2}{*}{ SP } & \multirow{2}{*}{$\mathrm{SP}+\mathrm{FOS}$} & \multirow{2}{*}{ NP } & \multirow{2}{*}{$\mathrm{NP}+\mathrm{FOS}$} & \multicolumn{3}{|c|}{ Two-way ANOVA } \\
\hline & & & & & FOS & Diet & Interaction \\
\hline Food intake, g & $147 \pm 3$ & $127 \pm 2$ & $146 \pm 5$ & $139 \pm 5$ & $<0.01$ & $<0.05$ & 0.38 \\
\hline Body weight gain, $g$ & $51 \pm 1$ & $46 \pm 3$ & $58 \pm 2$ & $51 \pm 2$ & $<0.05$ & 0.09 & 0.73 \\
\hline \multicolumn{8}{|l|}{ Cecum } \\
\hline Tissue weight, $\mathrm{g}$ & $0.47 \pm 0.01^{\mathrm{d}}$ & $1.25 \pm 0.05^{\mathrm{a}}$ & $0.81 \pm 0.03^{b}$ & $1.00 \pm 0.05^{\mathrm{c}}$ & $<0.01$ & $<0.05$ & $<0.01$ \\
\hline Contents, $\mathrm{g}$ & $1.71 \pm 0.05^{\mathrm{c}}$ & $4.27 \pm 0.26^{\mathrm{b}}$ & $5.34 \pm 0.08^{b}$ & $7.42 \pm 0.36^{\mathrm{a}}$ & $<0.01$ & $<0.01$ & $<0.01$ \\
\hline $\mathrm{pH}$ & $7.6 \pm 0.1^{\mathrm{a}}$ & $5.6 \pm 0.1^{\mathrm{d}}$ & $6.9 \pm 0.1^{\mathrm{b}}$ & $6.6 \pm 0.1^{c}$ & $<0.01$ & $<0.01$ & $<0.01$ \\
\hline \multicolumn{8}{|c|}{ Organic acids, $\mu \mathrm{mol} / \mathrm{g}$} \\
\hline Acetate & $33.2 \pm 2.4^{\mathrm{b}}$ & $18.2 \pm 1.5^{\mathrm{c}}$ & $80.6 \pm 2.4^{\mathrm{a}}$ & $88.1 \pm 2.7^{\mathrm{a}}$ & & & \\
\hline Propionate & $14.4 \pm 0.8^{\mathrm{a}}$ & $7.2 \pm 1.1^{\mathrm{b}}$ & $14.2 \pm 0.4^{\mathrm{a}}$ & $16.8 \pm 0.9^{\mathrm{a}}$ & & & \\
\hline$n$-Butyrate & $4.9 \pm 0.2^{\mathrm{b}}$ & $4.7 \pm 1.7^{\mathrm{b}}$ & $28.7 \pm 1.9^{\mathrm{a}}$ & $32.0 \pm 2.2^{\mathrm{a}}$ & & & \\
\hline SCFA & $52.6 \pm 3.4^{\mathrm{b}}$ & $28.9 \pm 2.9^{c}$ & $123.5 \pm 4.4^{\mathrm{a}}$ & $136.9 \pm 4.3^{\mathrm{a}}$ & & & \\
\hline Succinate & $7.3 \pm 1.6^{\mathrm{b}}$ & $20.4 \pm 3.3^{\mathrm{a}}$ & $0.0 \pm 0.0^{\mathrm{b}}$ & $0.4 \pm 0.4^{\mathrm{b}}$ & & & \\
\hline Lactate & $0.0 \pm 0.0^{b}$ & $38.0 \pm 6.3^{\mathrm{a}}$ & $0.0 \pm 0.0^{\mathrm{b}}$ & $0.0 \pm 0.0^{\mathrm{b}}$ & & & \\
\hline \multicolumn{8}{|c|}{ Bacteria count, log copies/g } \\
\hline Total bacteria & $12.8 \pm 0.1$ & $13.1 \pm 0.1$ & $13.6 \pm 0.1$ & $13.7 \pm 0.1$ & 0.08 & $<0.01$ & 0.40 \\
\hline Lactobacilli & $9.2 \pm 0.2^{\mathrm{b}}$ & $10.9 \pm 0.1^{\mathrm{a}}$ & $10.8 \pm 0.1^{\mathrm{a}}$ & $10.8 \pm 0.1^{\mathrm{a}}$ & $<0.01$ & 0.39 & $<0.01$ \\
\hline Bifidobacteria & $8.6 \pm 0.3^{b}$ & $11.8 \pm 0.1^{\mathrm{a}}$ & $8.8 \pm 0.1^{\mathrm{b}}$ & $8.7 \pm 0.1^{\mathrm{b}}$ & $<0.01$ & $<0.01$ & $<0.01$ \\
\hline \multicolumn{8}{|c|}{ Cecal mucosa } \\
\hline \multicolumn{8}{|c|}{ Gene expression, relative value } \\
\hline IIgR & $1.0 \pm 0.2^{\mathrm{b}}$ & $2.1 \pm 0.2^{\mathrm{a}}$ & $1.0 \pm 0.2^{\mathrm{b}}$ & $0.7 \pm 0.1^{\mathrm{b}}$ & $<0.05$ & $<0.01$ & $<0.01$ \\
\hline$I F N-\gamma$ & $1.0 \pm 0.1^{\mathrm{b}}$ & $2.0 \pm 0.2^{\mathrm{a}}$ & $1.0 \pm 0.2^{\mathrm{b}}$ & $1.1 \pm 0.3^{\mathrm{b}}$ & $<0.05$ & $<0.05$ & $<0.01$ \\
\hline$I L-4$ & $1.0 \pm 0.2$ & $0.9 \pm 0.1$ & $1.0 \pm 0.1$ & $0.9 \pm 0.3$ & $<0.05$ & 0.32 & 0.32 \\
\hline $\mathrm{IL}-10$ & $1.0 \pm 0.1^{\mathrm{b}}$ & $1.7 \pm 0.1^{\mathrm{a}}$ & $1.0 \pm 0.1^{\mathrm{b}}$ & $0.7 \pm 0.1^{\mathrm{b}}$ & 0.12 & $<0.01$ & $<0.01$ \\
\hline TNF- $\alpha$ & $1.0 \pm 0.2^{\mathrm{b}}$ & $4.2 \pm 0.8^{\mathrm{a}}$ & $1.0 \pm 0.1^{b}$ & $0.7 \pm 0.1^{b}$ & $<0.01$ & $<0.01$ & $<0.01$ \\
\hline
\end{tabular}

Values are means \pm SE, $n=6$. A two-way ANOVA was used to assess the effects of diet, time of exposure, and interactions between diet and time. When the interaction was significant, further multiple comparisons were performed with the TukeyKramer post hoc test. When the data were not homogenous, the Kruskal-Wallis test was used to compare mean values and further multiple comparisons were performed with the Steel-Dwass test. Values without a common superscript letter differ, $p<0.05$. FOS, fructo-oligosaccharides; SP, semi-purified diet; NP, non-purified diet; $p I g R$, polymeric immunoglobulin receptor.

sis of bacterial translocation. The peritoneal cavity was swabbed, and samples of liver and spleen tissues were collected. The cecum was then removed and weighed. The cecal contents were homogenized and used for analyses of $\mathrm{pH}$, organic acids, and IgA concentrations. The cecal tissue was cut along the greater curvature and divided into two portions. One portion was used for mucosa preparation for the isolation of total RNA and successive gene expression analyses. The other portion was stored at $-80^{\circ} \mathrm{C}$ until measurement of MPO.

Experiment 2. Forty-four rats, weighing 130-150 g, were allocated to 4 groups of 11 rats each. Rats were allowed free access to water and one of the SP, SP+FOS, $\mathrm{NP}$ and NP+FOS for $10 \mathrm{~d}$. On day 7, 6 rats in each group received an oral administration of Cr-EDTA (20 mg per rat) at $1200 \mathrm{~h}$; feces were then collected every $6 \mathrm{~h}$ for $66 \mathrm{~h}$. All of the rats were killed by decapitation under pentobarbital anesthesia at $1300 \mathrm{~h}$ on day 10. For 5 rats in each group that did not previously receive Cr-EDTA, the ileo-cecal and ceco-colonic junctions were ligated, and the cecum was immediately removed. The whole cecum with its contents was frozen in dry ice/acetone and used for analysis of the epithelial mucus layer. The cecal contents were collected from the remaining 6 rats in each group and used for the analysis of microbiota composition based on $16 \mathrm{~S}$ ribosomal RNA genes sequences.

As a reference, we also analyzed microbiota composition of the cecal contents in rats fed the SP with or without $6 \%$ FOS for $56 \mathrm{~d}$. The study had been conducted previously and published in elsewhere (11), except for the results of microbial composition. Initial body weight and the strain of rats used in the previous study were virtually the same as for those used in the present study.

Cecal $p H$ and organic acids in fresh cecal contents. Cecal $\mathrm{pH}$ was measured using a compact $\mathrm{pH}$ meter (Model C-1, Horiba, Tokyo, Japan). Cecal organic acids were measured by a HPLC according to the internal standard method (19).

Measurements of urinary and fecal Cr-EDTA. Cr-EDTA was prepared according to the method of Binnerts et al. (20). Sample preparations for urinary (11) and fecal Cr-EDTA measurements (21) followed the methods described previously.

Bacterial translocation to MLN. MLNs were minced and homogenized in $0.5 \mathrm{~mL}$ of sterile brain-heart infusion broth (Wako Pure Chemical Industries, Ltd., Osaka, Japan) using a hand-held pestle homogenizer (Nippon 


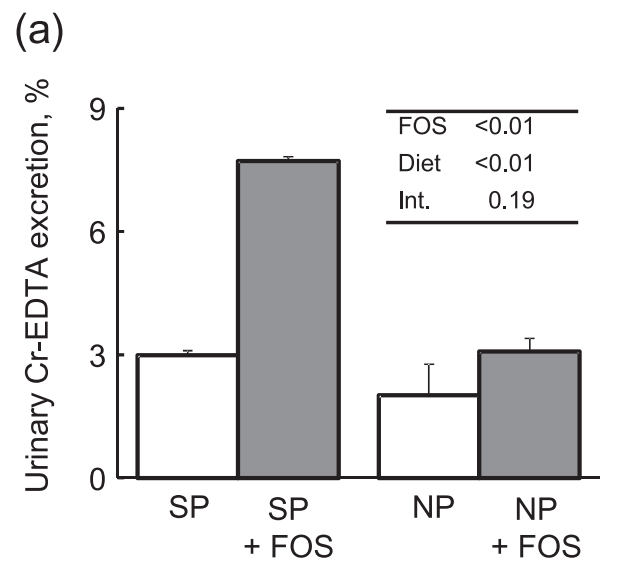

(b)

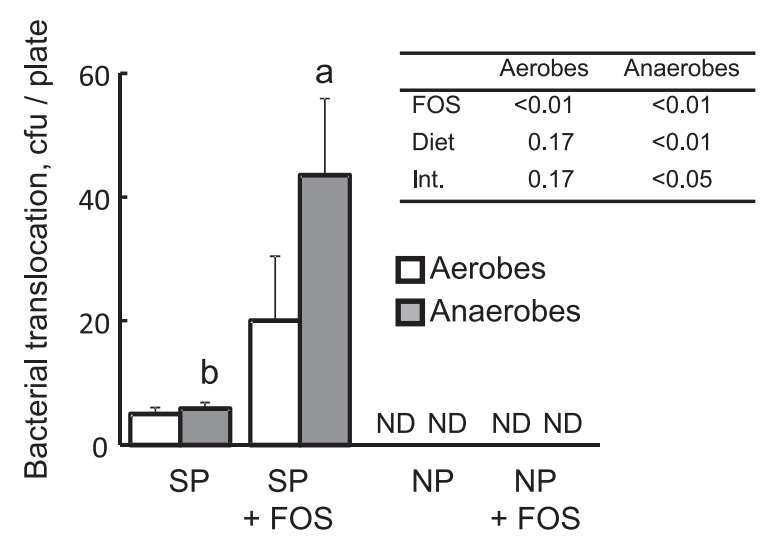

(c)

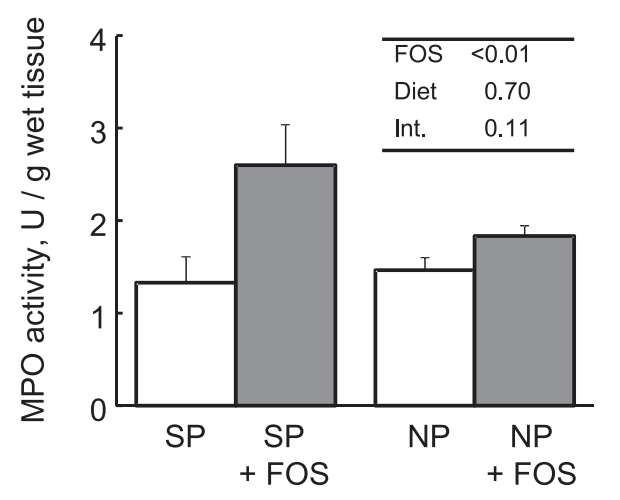

(d)

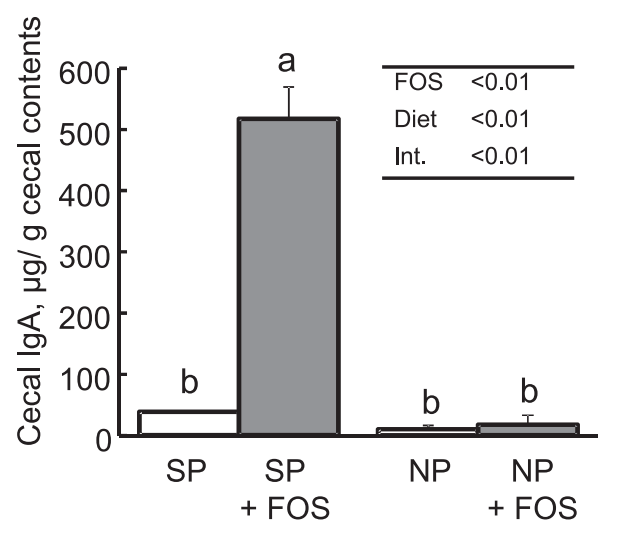

Genetics, Tokyo, Japan), and then $0.1 \mathrm{~mL}$ aliquots of the homogenate were spread onto brain-heart infusion agar plates (Wako Pure Chemical Industries, Ltd.), which were subsequently incubated under either aerobic or anaerobic conditions at $37^{\circ} \mathrm{C}$ for 24 or $48 \mathrm{~h}$. Liver and spleen tissue (approx. $50 \mathrm{mg}$ ) homogenates and peritoneal cavity swabs were also placed in sterile brain-heart infusion broth and treated in a similar manner as MLN. After incubation, colonies on the plates were counted.

Measurement of MPO. MPO was determined by the method of Bradley et al. (22). One unit of MPO activity was defined as that required to convert $1 \mu \mathrm{mol}$ of hydrogen peroxide to water per min at $25^{\circ} \mathrm{C}$.

IgA analysis in fresh cecal contents. Cecal IgA was determined by enzyme-linked immunosorbent assay as described previously (23).

RNA isolation and quantitative real-time polymerase chain reaction (RT-PCR) analysis. Total RNA isolation and quantitative RT-PCR were performed as previously described (23). The primer pairs and protocols for PCR of IFN- $\gamma$, polymeric immunoglobulin receptor ( $p I g R)$, IL-4, IL-10, TNF- $\alpha$ and 18 S rRNA have been previously reported (11). 18S rRNA was used as an endogenous reference gene. To confirm amplification specificity, the PCR products from each primer pair were subjected to a melting curve analysis. Gene expression was quantified using the comparative cycle threshold method (24), and the data were expressed relative to the control group.

Quantification of bifidobacteria, lactobacilli, and total bacteria in fresh cecal contents by RT-PCR. DNA was extracted from cecal contents using a fecal DNA isolation kit (Mo Bio Laboratories, Carlsbad, CA) according to the manufacturer's instructions. 16S rDNA fragments were amplified and detected using a Light Cycler nano (Roche, Bazel, Switzerland). Genus-specific Bifidobacterium (25) and Lactobacillus (26) primers and universal primers for bacteria (27) were used. For quantification, 16S rDNA fragments were amplified from the genomic DNA of B. animalis (JCM 1190T), L. murinus (JCM 1717T) or Escherichia coli DH5 $\alpha$ (Takara, Shiga, Japan), as described previously (23).

Analysis of the epithelial mucus layer. According to the

Fig. 1. Urinary Cr-EDTA excretion (a), bacterial translocation into MLNs (b), MPO activity in the cecal tissue (c), and cecal IgA concentrations (d) of male Wistar rats fed either SP or NP with or without $60 \mathrm{~g}$ of FOS/kg diet for $9 \mathrm{~d}$ (experiment 1). Values are means \pm SE, $n=6$. A two-way ANOVA was basically used to assess the effects of diet, time of exposure, and interactions between diet and time. When the interaction was significant, further multiple comparisons were performed with the TukeyKramer post hoc test. For cecal IgA concentrations and bacterial translocation, the nonparametric Kruskal-Wallis test was used to compare mean values because of unequal variance. Further multiple comparisons were performed with the Steel-Dwass test. Values without a common superscript letter differ, $p<0.05$. CFU, colony forming units; FOS, fructo-oligosaccharides; MLN, mesenteric lymph node; MPO, myeloperoxidase; ND, not detected (detection limit was one CFU/plate); NP, non-purified diet; SP, semi-purified diet. 
SP

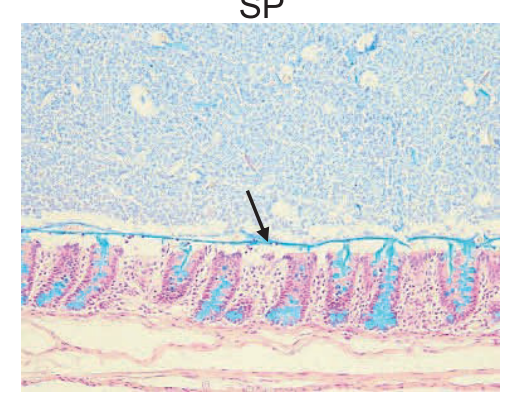

NP

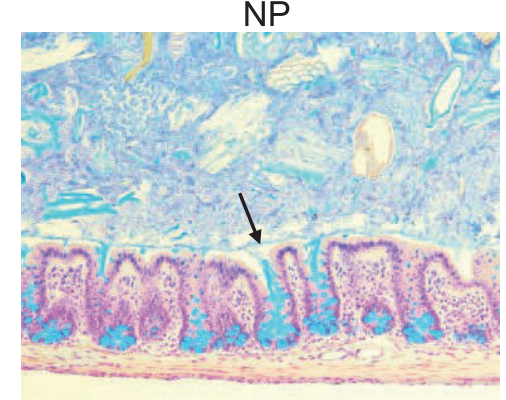

$\mathrm{SP}+\mathrm{FOS}$

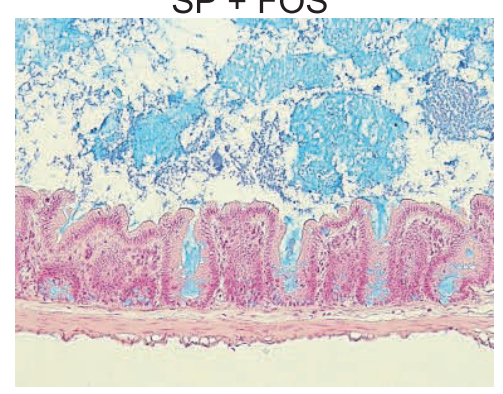

$\mathrm{NP}+\mathrm{FOS}$

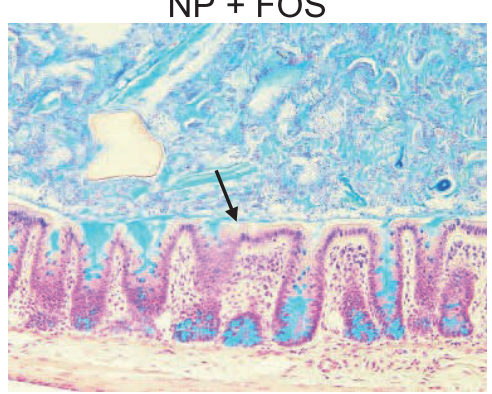

Fig. 2. Cecum thin sections stained with Alcian blue prepared from male Wistar rats fed either SP or NP with or without $60 \mathrm{~g}$ of FOS/kg diet for $10 \mathrm{~d}$ (experiment 2). Black arrows on the photos indicate mucus layers between the epithelium and cecum digesta. FOS, fructo-oligosaccharides; NP, non-purified diet; SP, semi-purified diet. Magnification, ×100.

method of Tsukahara et al. (28), the frozen cecum tissues were cut into cross-sections approximately 10-mm thick at the middle portion of the cecum with a frozen saw. The frozen section was fixed in Carnoy solution at room temperature overnight. Care was taken to avoid constriction of the tissue due to over-fixation. The fixed specimens were immersed in $80 \%$ ethanol for an additional $24 \mathrm{~h}$ at room temperature and then embedded in paraffin. Cross-sections of $3-\mu \mathrm{m}$ thickness were prepared from the paraffin-embedded samples and stained with Alcian blue ( $\mathrm{pH} 2.5$ ) and then counterstained with Kernechtrot.

Microbial composition analysis. Bacterial DNA in the cecal contents was extracted using QIIAMP Fast Stool Kit (Qiagen, Hilden, Germany) by following the manufacturer's protocol. The V3-V4 region of the bacterial 16S rRNA gene was amplified and indexed by following Illumina's 16S Metagenomic Sequencing Library Preparation protocol (Illumina, San Diego, CA). The libraries were sequenced on the MiSeq sequencer using a reagent v3 cartridge and 600 cycles (Illumina). Demultiplexing and operational taxonomic unit (OTU) picking of 16S rRNA genes sequences were done in Quantitative Insights Into Microbial Ecology, v.1.9.1 (QIIME) (29). OTUs sharing $97 \%$ identity were clustered using the CDHIT algorithm (30). After alignment of the sequences and removal of chimeras, the representative sequences were classified through the NCBI taxonomy database. Rarefaction curves ( $\alpha$-diversity curves) and a principal coordinate analysis plot based on weighted UniFrac ( $\beta$-diversity) were also generated with the use of QIIME.

Statistical analyses. Data were expressed as means \pm SE. Statistical analyses were carried out using JMP8.0.1 software (SAS Institute, Cary, NC). Variance homogene- ity was examined by the Bartlett test. In experiments 1 and 2, two-way ANOVA was basically used to assess the effects of diet, FOS, and interactions between them. When the interaction was significant, further multiple comparisons were performed with the Tukey-Kramer post hoc test. For cecal concentrations of organic acids and $\operatorname{IgA}$, and bacterial translocation (experiment 1 ), the nonparametric Kruskal-Wallis test was used to compare mean values because of unequal variance. Further multiple comparisons were performed with the Steel-Dwass test. The data for fecal Cr-EDTA excretion over the time (experiment 2) was further analyzed by 2-way repeated measures ANOVA. As for $\beta$-diversity (experiment 2), analysis of similarity (ANOSIM) with 999 permutations was used to detect statistical significances between microbial compositions in different dietary groups. Statistical significance was accepted at $p<0.05$.

\section{RESULTS}

\section{Experiment 1}

Food intakes were significantly affected by both FOS and diet factors, whereas only the FOS factor was significant in body weight gain (Table 2). The interactions between FOS and diet factors were significant in cecal variables including tissue and content weights, and $\mathrm{pH}$ values. The cecal tissue weights were greater in the order of $\mathrm{SP}+\mathrm{FOS}>\mathrm{NP}+\mathrm{FOS}>\mathrm{NP}>\mathrm{SP}$, and the cecal content weights were greater in the order of $\mathrm{NP}+\mathrm{FOS}>$ $\mathrm{NP}>\mathrm{SP}+\mathrm{FOS}>\mathrm{SP}$, whereas the $\mathrm{pH}$ values were lower in the order of $\mathrm{SP}+\mathrm{FOS}>\mathrm{NP}+\mathrm{FOS}>\mathrm{NP}>\mathrm{SP}$. All of these differences were significant. Cecal acetate, n-butyrate, and total SCFA concentrations were greater in the NP and NP+FOS than in the SP and SP+FOS. Cecal propionate concentrations were lower in SP than in the 


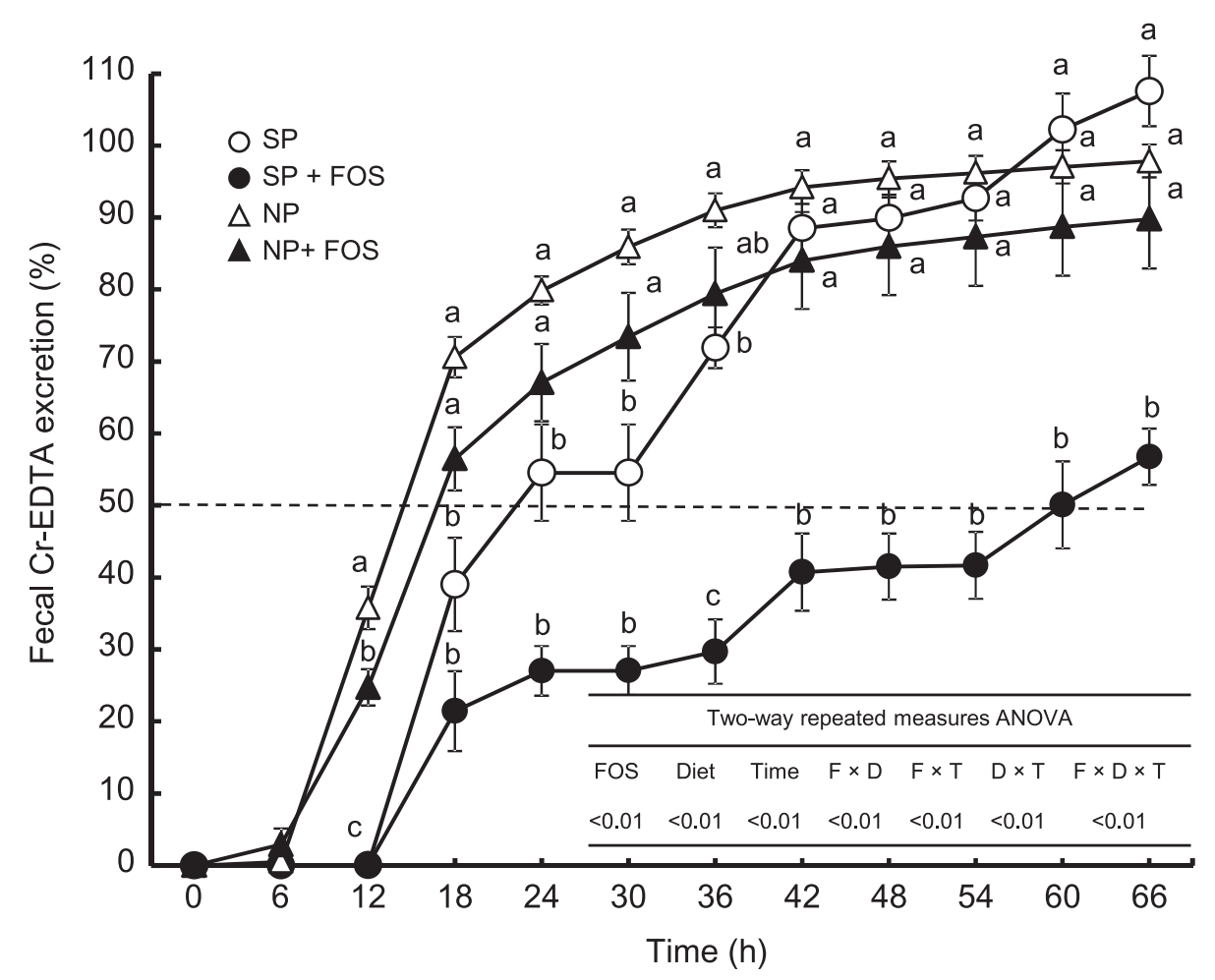

Fig. 3. Fecal Cr-EDTA excretion in male Wistar rats fed either SP and NP with or without $60 \mathrm{~g}$ of FOS/kg diet for $10 \mathrm{~d}$ (experiment 2). Values are means \pm SE, $n=6$. A two-way repeated measures ANOVA was used to assess the effects of FOS, diet, time of exposure, and interactions between them. When the interaction was significant, further multiple comparisons were performed with the Tukey-Kramer post hoc test. Values without a common superscript letter differ, $p<0.05$. FOS, fructo-oligosaccharides; NP, non-purified diet; SP, semi-purified diet.

others, whereas cecal succinate concentrations were greater in SP than in the others. The number of total bacteria was affected solely by diet factors. The interactions between FOS and diet factors were significant in the number of both lactobacilli and bifidobacteria. The numbers of lactobacilli were higher in SP+FOS, NP and $\mathrm{NP}+\mathrm{FOS}$ than in SP, while those of bifidobacteria were higher than in the others solely in SP+FOS. As for the cecal mRNA expression, the interactions between FOS and diet factors were significant in $p I g R, I F N-\gamma, I L-10$, and TNF- $\alpha$, and the expression of these were higher in $\mathrm{SP}+\mathrm{FOS}$ than in the others. Only the FOS factor was significant in $I L-4$.

Urinary Cr-EDTA excretions were significantly affected by both FOS and diet factors (Fig. 1a). Bacterial translocation into MLN in SP+FOS was 5 to 10 times greater than in SP under aerobic and anaerobic conditions (Fig. 1b). No bacterial translocation was detected in NP or NP+FOS. With respect to bacterial cultures, liver and spleen samples and peritoneal cavity swabs were all negative. MPO activities in the cecum were affected only by the FOS factor (Fig. 1c). Cecal IgA concentrations in $\mathrm{SP}+\mathrm{FOS}$ was more than 10 fold greater than in the others (Fig. 1d).

Experiment 2

Trends similar to those observed with experiment 1 were also observed in experiment 2 with respect to food intakes (SP, $157 \pm 4 \mathrm{~g}$; SP+FOS, $144 \pm 4 \mathrm{~g}$; NP, $152 \pm 3 \mathrm{~g}$; $\mathrm{NP}+\mathrm{FOS}, 153 \pm 1 \mathrm{~g}$ for $10 \mathrm{~d}$ ), and body weight gains
(SP, $58 \pm 1 \mathrm{~g}$; SP+FOS, $56 \pm 4 \mathrm{~g}$; NP, $52 \pm 2 \mathrm{~g}$; NP+FOS, $53 \pm 2 \mathrm{~g}$ for $10 \mathrm{~d}$ ). Body weight gains were affected neither by FOS nor by diet. Significant interaction between FOS and diet were observed in food intakes, and that of SP+FOS was significantly lower than those of the others, which did not differ. Alcian blue staining of crosssections prepared from the cecum with its contents enabled visualization of the mucus layer between the epithelium and cecal contents in SP, and also, though somewhat blurred in NP and NP+FOS. The mucus layer in SP+FOS, however, completely disappeared (Fig. 2, individual photos are shown in Supplemental Online Material, Figs. S1 and S2). Fecal Cr-EDTA excretions were significantly affected by diet, FOS and time factors, and the interactions among them were all significant (Fig. 3). Between $18 \mathrm{~h}$ and $30 \mathrm{~h}$, fecal Cr-EDTA excretions were greater in the order of NP, NP+FOS $>$ SP, SP+FOS. Between $36 \mathrm{~h}$ and $66 \mathrm{~h}$, fecal Cr-EDTA excretions in $\mathrm{SP}+\mathrm{FOS}$ were significantly lower than in the others. The time for $50 \%$ fecal excretion of $\mathrm{Cr}$ EDTA, when calculated from the individual excretion curves, was 2 to 3 times significantly longer in SP+FOS $(62 \pm 2 \mathrm{~h})$ than in the others (SP, $30 \pm 4 \mathrm{~h}$; NP, $18 \pm 1 \mathrm{~h}$; $\mathrm{NP}+\mathrm{FOS}, 21 \pm 1 \mathrm{~h}$ ), which did not differ.

The V3-V4 region of bacterial 16S rRNA obtained from the cecal contents of 24 rats was amplified by PCR and sequenced on the Miseq platform. A total of $1,169,345$ quality-filtered sequences was obtained. Each sample was covered by an average of 48,722 
reads/rat (31,336-49,558 reads). These reads were clustered into 3,499 unique OTUs with an average of 828 OTUs/rat (469-1,430 OTUs). Alpha diversity analysis of entire OTUs was performed after rarefaction to 30,100 sequences/sample (maximum sampling depth) for estimation of species richness (Fig. 4a). Two-

(a)

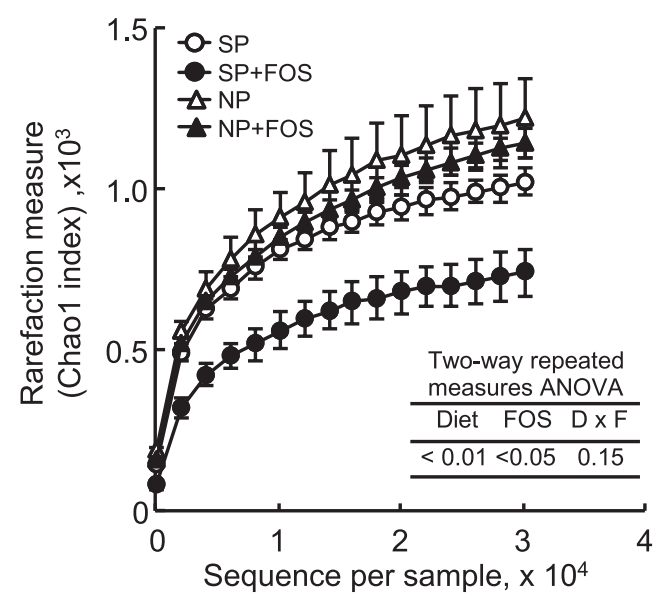

(b)

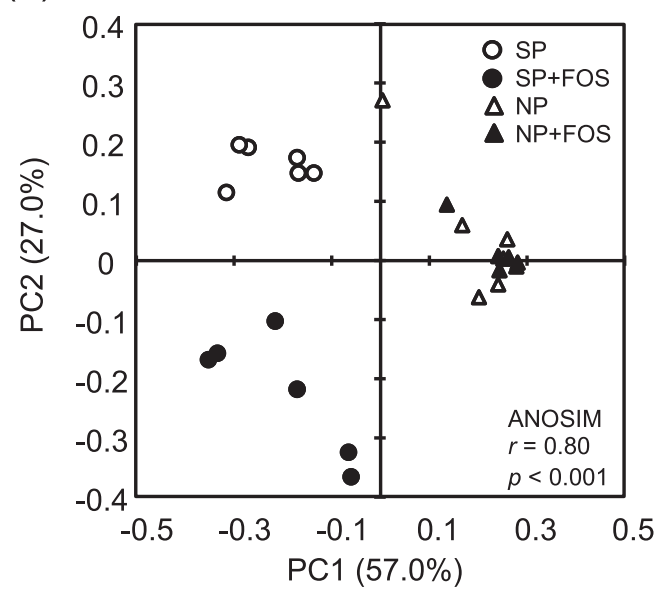

(c)

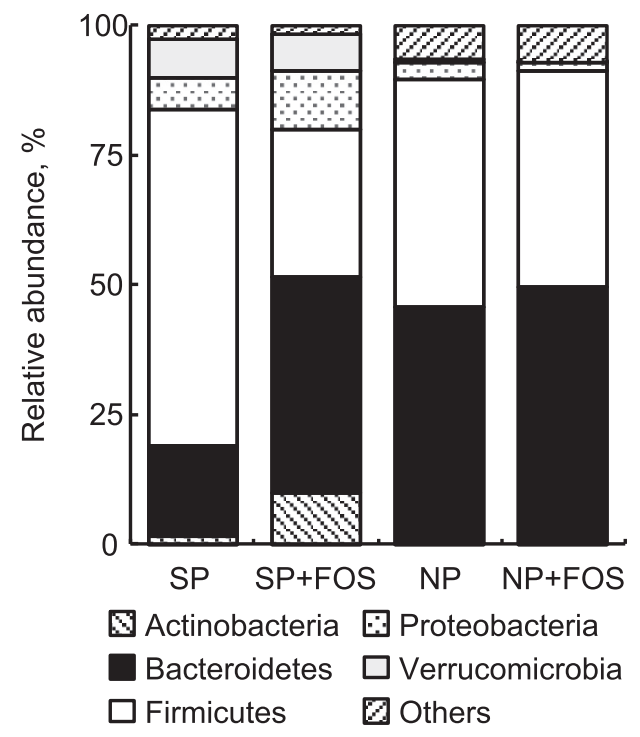
diet. way repeated measures ANOVA on rarefaction curves showed that both diet and FOS factors affect species richness. Comparison between the groups at each sampling size of sequence showed that species richness in $\mathrm{SP}+\mathrm{FOS}$ was far less than those in the others $(p<0.01)$. In weighed UniFrac PCoA, the first principal coordinate (PC1) captured $57.0 \%$ of the inter-sample variance and revealed a distinction between the microbiota of SP and $\mathrm{SP}+\mathrm{FOS}$ versus NP and NP+FOS (Fig. 4b). The second principal coordinate (PC2) captured $27.0 \%$ of the intersample variance and revealed a distinction between the microbiota of SP and SP+FOS. The differences among the dietary groups were significant $(r=0.80, p<0.001)$. The vast majority of sequences in each group (93-98\%) was assigned to five phyla: Firmicutes, Bacteroidetes, Proteobacteria, Verrucomicrobia, and Actinobacteria (Fig. 4c). Firmicutes was the most abundant phylum in SP $(65 \pm 2 \%)$, whereas Bacteroidetes was the most abundant phylum in SP+FOS (42 $\pm 5 \%)$. Firmicutes $(44 \pm 6 \%$ and $42 \pm 3 \%$ ) and Bacteroidetes (45 $\pm 5 \%$ and $49 \pm 3 \%$ ) evenly dominated in NP and NP+FOS with low representation of Proteobacteria $(2 \pm 0 \%$ and $3 \pm 1 \%)$, Actinobacteria $(<0.1 \%)$ and Verrucomicrobia $(<0.1 \%)$. Proteobacteria, Actinobacteria, and Verrucomicrobia have a modest population in SP $(6 \pm 1 \%, 2 \pm 0 \%$, and $7 \pm 1 \%)$ and SP+FOS $(10 \pm 2 \%, 10 \pm 1 \%$, and $7 \pm 1 \%)$. Moreover, Proteobacteria, Actinobacteria, and Verrucomicrobia in SP+FOS were largely composed of Parasutterella, Bifidobacterium, and Akkermansia at the genus level.

Microbial composition in SP and SP+FOS after 56 d-feeding study is shown in Supplemental Online Material, Fig. S3 as a reference. Compared with the results of the $9 \mathrm{~d}$-feeding study (Fig. 4), the $\alpha$-diversity was still largely lower in FOS + SP than in SP, while the $\beta$-diversity of the cecal microbiota between the two dietary groups became closer, but the difference was still significant. At the phyla level, the relative abundances of Firmicutes and Bacteroidetes became similar between the two dietary groups.

\section{DISCUSSION}

In accordance with our previous studies $(11,31)$, FOS supplementation induced a very acidic fermentation in the cecum due to the accumulation of lactate and succinate in rats fed SP for $9 \mathrm{~d}$. FOS ingestion was also accompanied by simultaneous significant increases in gut

Fig. 4. Rarefaction curves ( $\alpha$-diversity curves) (a), principal coordinate analysis plot based on weighted UniFrac ( $\beta$-diversity) (b), and microbial compositions at the phyla level (c) of the cecal contents in male Wistar rats fed either SP or NP with or without $60 \mathrm{~g}$ of FOS/ $\mathrm{kg}$ diet for $10 \mathrm{~d}$ (experiment 2). Values are means \pm SE, $n=6$. A two-way repeated measures ANOVA was used to assess the effects of FOS, diet, time of exposure, and interactions between them ( $\alpha$-diversity curves). ANOSIM was used to detect statistical significances between microbial compositions in different dietary groups ( $\beta$-diversity). ANOSIM, analysis of similarity; FOS, fructo-oligosaccharides; NP, non-purified diet; PC1, the first principal coordinate; PC2, the second principal coordinate; SP, semi-purified 
permeability (as assessed by urinary Cr-EDTA excretion and translocation of bacteria into MLNs) and myeloperoxidase activities (as a marker of neutrophil accumulation), and the expressions of inflammatory cytokine genes in the cecal tissues. In contrast, the fermentation pattern in rats fed NP was characterized by the production toward more SCFA and less succinate and lactate, irrespective of supplemental FOS in the diet. Consequently, all of the FOS-induced alterations related to gut permeability and mucosal inflammatory responses were totally cancelled in rats fed NP. Clearly, the impact of FOS on the gut mucosa quite differs between rats fed SP and NP. Regarding this difference, most of the previous publications regarding a positive effect of FOS on the gut barrier function (7-9) have been conducted in animals receiving NP, which may produce much more abundant large bowel SCFAs than in those receiving an AIN-based SP. Among SCFAs, butyrate in particular might promote secretion of mucin from goblet cells (32) and restoration of the mucus layer on the epithelial surface in the cecum. Recently, bacteria-derived SCFAs have been shown to stabilize the hypoxia-inducible factor, a transcription factor coordinating barrier protection through the colonic epithelial $\mathrm{O}_{2}$ consumption (33). Thus, the different fermentation patterns of FOS may be indicative of the different outcomes on the gut-barrier function between rats fed SP and NP in the present study.

FOS ingestion greatly increased cecal IgA concentrations in rats fed SP, but the effects disappeared in rats fed NP. Previous study clearly showed that the early elevation of cecal IgA by ingestion of FOS-supplemented SP represented a physiological response that acted to maintain gut mucosa homeostasis against the increase in gut permeability and the related mucosal inflammation (11). This is because the expression of pIgR, a critical molecule to convey polymeric IgA across the intestinal epithelium to the mucosal surface, is upregulated by a number of proinflammatory cytokines and subsequent activation of NF- $\kappa \mathrm{B}$ and STAT pathways (34, 35). Accordingly, the impact of FOS on the cecal IgA secretion quite differs in rats fed SP and NP; no mucosal inflammation occurred in the latter. Further, both quantification of bifidobacteria by RT-PCR and analysis of $16 \mathrm{~S}$ rRNA gene sequences corroborated the bifidogenic property of FOS in rats fed SP, but this is not the case in rats fed NP. These diet-specific effects of FOS were also manifest in the evaluation of dextran sodium sulfate-induced rat colitis in which FOS supplementation severely deteriorated the colitis in rats fed SP $(36,37)$, but not in rats fed NP $(37,38)$. Therefore, when FOS effects are discussed, caution must be exercised to define which physiologic effects are exerted under which diet conditions.

The mechanism(s) by which supplemental FOS in the SP induces increased gut permeability in the early stage of FOS ingestion has not been fully elucidated. The most probable explanation, however, is disintegration of the mucus layer in the cecum that may prevent direct contact between commensal bacteria and the epithelial surface, as indicated by the present and the previous studies (11). Tsukahara et al. reported a similar observation in rats fed 10\% FOS supplemental SP (28). Such a disruption of the mucus layer was not observed in rats fed the FOS-supplemented NP. Lidell et al. demonstrated that an autocatalytic (non-enzymatic) cleavage in the C-terminal of Muc2 mucin, a major part of the mucous layer, occurred at an Asp-Pro bond in a time-dependent manner at a pH below 6.0 (39). In addition to the very acidic fermentation in the cecum, the present study also showed that intestinal transit time of the luminal contents in rats fed the FOS-supplemented SP was 2-3 times longer than in those fed the other diets. Sakaguchi et al. reported that $10 \%$ FOS supplementation significantly prolonged mean retention time of the luminal contents in rats fed SP, and that the mean retention time was strongly associated with enlargement of the cecum (40). It is therefore plausible to assume that "stasis" of the very acidic contents in the cecum may accelerate perturbation of the mucus layer in the cecum, leading to the increased gut permeability in rats fed the FOSsupplemented SP.

The drop in cecal $\mathrm{pH}$ due to enhanced fermentation of FOS inhibits the growth of acid-sensitive bacteria, and provides a growth-advantage of more acid-resistant bacteria including lactic acid-producing bacteria such as lactobacilli and bifidobacteria (16). As a consequence in the present study, FOS supplementation to SP induced a lower $\alpha$-diversity and a distinct separation on the $\beta$-diversity compared with SP, but this was not the case in NP with or without FOS supplementation. Dysfermentation with the accumulation of succinate and lactate, and lowered diversity of the cecal microbiota may represent a certain aspect of dysbiosis in rats fed the FOS-supplemented SP. Under an extreme fluctuation of microbiota composition, bacterial translocation to MLN could be stimulated because commensal bacteria themselves function as a barrier (41). However, even after prolonged ingestion of FOS ( $56 \mathrm{~d}$ ) in which gut permeability returned to the normal level and bacterial translocation into MLN was negligible (11), $\alpha$-diversity in rats fed the FOS-supplemented SP was still lower than in those fed SP, and the $\beta$-diversity of the cecal microbiota differed between the two dietary groups (Supplemental Online Material, Fig. S3). Liu et al. also reported a lower species richness in rats fed $10 \%$ short-chain fructan- or inulin-supplemented SP for $4 \mathrm{wk}$ (42). These findings may suggest that the lowered diversity of microbiota per se is not necessary associated with transitional increases in the gut permeability or bacterial translocation in rats fed the FOS-supplemented SP.

In conclusion, the impact of FOS on the gut permeability, bacterial translocation, mucosal inflammation, and luminal IgA secretion in the cecum quite differs between rats fed SP and NP. From the findings of comparative study using SP and NP, it is assumed that increased gut permeability and mucosal inflammation in the cecum in rats fed the FOS-supplemental SP are evoked by the disruption of the mucus layer due to stasis of very acidic luminal contents with the accumulation of lactate and succinate. 
Supporting information

Supplemental Online Material is available on J-STAGE.

\section{REFERENCES}

1) Guarner F. 2007. Studies with inulin-type fructans on intestinal infections, permeability, and inflammation. J Nutr 137: 2568S-2571S.

2) Colomé G, Sierra C, Blasco J, García MV, Valverde E, Sánchez E. 2007. Intestinal permeability in different feedings in infancy. Acta Paediatr 96: 69-72.

3) Ten Bruggencate SJ, Bovee-Oudenhoven IM, LettinkWissink ML, Van der Meer R. 2003. Dietary fructo-oligosaccharides dose-dependently increase translocation of salmonella in rats. J Nutr 133: 2313-2318.

4) Schepens MA, Rijnierse A, Schonewille AJ, Vink C, Brummer RJ, Willemsen LE, van der Meer R, BoveeOudenhoven IM. 2010. Dietary calcium decreases but short-chain fructo-oligosaccharides increase colonic permeability in rats. Br J Nutr 104: 1780-1786.

5) Barrat E, Michel C, Poupeau G, David-Sochard A, Rival M, Pagniez A, Champ M, Darmaun D. 2008. Supplementation with galactooligosaccharides and inulin increases bacterial translocation in artificially reared newborn rats. Pediatr Res 64: 34-39.

6) Petersen A, Heegaard PM, Pedersen AL, Andersen JB, Sørensen RB, Frøkiaer H, Lahtinen SJ, Ouwehand AC, Poulsen M, Licht TR. 2009. Some putative prebiotics increase the severity of Salmonella enterica serovar Typhimurium infection in mice. BMC Microbiol 9: 245.

7) Fontaine N, Meslin JC, Lory S, Andrieux C. 1996. Intestinal mucin distribution in the germ-free rat and in the heteroxenic rat harbouring a human bacterial flora: effect of inulin in the diet. Br J Nutr 75: 881-892.

8) Kleessen B, Hartmann L, Blaut M. 2003. Fructans in the diet cause alterations of intestinal mucosal architecture, released mucins and mucosa-associated bifidobacteria in gnotobiotic rats. Br J Nutr 89: 597-606.

9) Kleessen B, Blaut M. 2005. Modulation of gut mucosal biofilms. Br J Nutr 93 (Suppl 1): S35-40.

10) Johnson-Henry KC, Pinnell LJ, Waskow AM, Irrazabal T, Martin A, Hausner M, Sherman PM. 2014. Short-chain fructo-oligosaccharide and inulin modulate inflammatory responses and microbial communities in Caco2-bbe cells and in a mouse model of intestinal injury. J Nutr 144: 1725-1733.

11) Genda T, Sasaki Y, Kondo T, Hino S, Nishimura N, Tsukahara T, Sonoyama K, Morita T. 2017. Fructo-oligosaccharide-induced transient increases in cecal immunoglobulin A concentrations in rats are associated with mucosal inflammation in response to increased gut permeability. J Nutr 147: 1900-1908.

12) Umesaki Y, Yajima T, Yokokura T, Mutai M. 1979. Effect of organic acid absorption on bicarbonate transport in rat colon. Pflugers Arch 14(379): 43-47.

13) Morita T, Kasaoka S, Ohhashi A, Ikai M, Numasaki Y, Kiriyama S. 1998. Resistant proteins alter cecal shortchain fatty acid profiles in rats fed high amylose cornstarch. J Nutr 128: 1156-1164.

14) Morita T, Kasaoka S, Hase K, Kiriyama S. 1999. OligoL-methionine and resistant protein promote cecal butyrate production in rats fed resistant starch and fructooligosaccharide. J Nutr 129: 1333-1339.

15) Morita T, Kasaoka S, Hase K, Kiriyama S. 1999. Psyllium shifts the fermentation site of high-amylose cornstarch toward the distal colon and increases fecal buty- rate concentration in rats. J Nutr 129: 2081-2087.

16) Vogt L, Meyer D, Pullens G, Faas M, Smelt M, Venema K, Ramasamy U, Schols HA, De Vos P. 2015. Immunological properties of inulin-type fructans. Crit Rev Food Sci Nutr 55: 414-436.

17) American Institute of Nutrition. 1977. Report of the American Institute of Nutrition ad hoc committee on standards for nutritional studies. J Nutr 107: 1340-1348.

18) Prosky L, Asp NG, Furda I, DeVries JW, Schweizer TF, Harland BF. 1985. Determination of total dietary fiber in foods and food products: collaborative study. J Assoc Off Anal Chem 68: 677-679.

19) Hoshi S, Sakata T, Mikuni K, Hashimoto H, Kimura S. 1994. Galactosylsucrose and xylosylfructoside alter digestive tract size and concentrations of cecal organic acids in rats fed diets containing cholesterol and cholic acid. J Nutr 124: 52-60.

20) Binnerts W, Van het Klooster A, Frens A. 1968. Soluble chromium indicator measured by atomic absorption in digestion experiments. Vet Rec 82: 470.

21) Kondo T, Handa K, Genda T, Hino S, Hamaguchi N, Morita T. 2017. Digestion-resistant dextrin derivatives are moderately digested in the small intestine and contribute more to energy production than predicted from large-bowel fermentation in rats. J Nutr 147: 330-336.

22) Bradley PP, Priebat DA, Christensen RD, Rothstein G. 1982. Measurement of cutaneous inflammation: estimation of neutrophil content with an enzyme marker. J Invest Dermatol 78: 206-209.

23) Ito H, Takemura N, Sonoyama K, Kawagishi H, Topping DL, Conlon MA, Morita T. 2011. Degree of polymerization of inulin-type fructans differentially affects number of lactic acid bacteria, intestinal immune functions, and immunoglobulin A secretion in the rat cecum. J Agric Food Chem 59: 5771-5778.

24) Heid CA, Stevens J, Livak KJ, Williams PM. 1996. Real time quantitative PCR. Genome Res 6: 986-994.

25) Rinttilä T, Kassinen A, Malinen E, Krogius L, Palva A. 2004. Development of an extensive set of 16S rDNAtargeted primers for quantification of pathogenic and indigenous bacteria in faecal samples by real-time PCR. J Appl Microbiol 97: 1166-1177.

26) Byun R, Nadkarni MA, Chhour KL, Martin FE, Jacques NA, Hunter N. 2004. Quantitative analysis of diverse Lactobacillus species present in advanced dental caries. J Clin Microbiol 42: 3128-3136.

27) Makker HPS, McSweeney CS, eds. 2010. Methods in Gut Microbial Ecology for Ruminants. Springer, Dordrecht.

28) Tsukahara T, Iwasaki Y, Nakayama K, Ushida K. 2002. An improved technique for the histological evaluation of the mucus-secreting status in rat cecum. J Nutr Sci Vitaminol 48: 311-314.

29) Caporaso JG, Kuczynski J, Stombaugh J, Bittinger K, Bushman FD, Costello EK, Fierer N, Peña AG, Goodrich JK, Gordon JI, Huttley GA, Kelley ST, Knights D, Koenig JE, Ley RE, Lozupone CA, McDonald D, Muegge BD, Pirrung M, Reeder J, Sevinsky JR, Turnbaugh PJ, Walters WA, Widmann J, Yatsunenko T, Zaneveld J, Knight R. 2010. QIIME allows analysis of high-throughput community sequencing data. Nat Methods 7: 335-336.

30) Edgar RC. 2010. Search and clustering orders of magnitude faster than BLAST. Bioinformatics 26: 2460-2461.

31) Komura M, Fukuta T, Genda T, Hino S, Aoe S, Kawagishi H, Morita T. 2014. A short-term ingestion of fructo-oli- 
gosaccharides increases immunoglobulin A and mucin concentrations in the rat cecum, but the effects are attenuated with the prolonged ingestion. Biosci Biotechnol Biochem 78: 1592-1602.

32) Sakata T, Setoyama H. 1995. Local stimulatory effect of short-chain fatty acids on the mucus release from the hindgut mucosa of rats (Rattus norvegicus). Comp Biochem Physiol A Physiol 111: 429-432.

33) Kelly CJ, Zheng L, Campbell EL, Saeedi B, Scholz CC, Bayless AJ, Wilson KE, Glover LE, Kominsky DJ, Magnuson A, Weir TL, Ehrentraut SF, Pickel C, Kuhn KA, Lanis JM, Nguyen V, Taylor CT, Colgan SP. 2015. Crosstalk between microbiota-derived short-chain fatty acids and intestinal epithelial HIF augments tissue barrier function. Cell Host Microbe 17: 662-671.

34) Johansen FE, Brandtzaeg P. 2004. Transcriptional regulation of the mucosal IgA system. Trends Immunol 25: 150-157.

35) Kaetzel CS. 2005. The polymeric immunoglobulin receptor: bridging innate and adaptive immune responses at mucosal surfaces. Immunol Rev 206: 83-99.

36) Moreau NM, Martin LJ, Toquet CS, Laboisse CL, Nguyen PG, Siliart BS, Dumon HJ, Champ MM. 2003. Restoration of the integrity of rat caeco-colonic mucosa by resistant starch, but not by fructo-oligosaccharides, in dextran sulfate sodium-induced experimental colitis. $\mathrm{Br}$ J Nutr 90: 75-85.

37) Goto H, Takemura N, Ogasawara T, Sasajima N, Wata- nabe J, Ito H, Morita T, Sonoyama K. 2010. Effects of fructo-oligosaccharide on DSS-induced colitis differ in mice fed nonpurified and purified diets. J Nutr 140: 2121-2127.

38) Videla S, Vilaseca J, Antolín M, García-Lafuente A, Guarner F, Crespo E, Casalots J, Salas A, Malagelada JR. 2001. Dietary inulin improves distal colitis induced by dextran sodium sulfate in the rat. Am J Gastroenterol 96: 1486-1493.

39) Lidell ME, Johansson ME, Hansson GC. 2003. An autocatalytic cleavage in the $\mathrm{C}$ terminus of the human MUC2 mucin occurs at the low $\mathrm{pH}$ of the late secretory pathway. J Biol Chem 278: 13944-13951.

40) Sakaguchi E, Sakoda C, Toramaru Y. 1998. Caecal fermentation and energy accumulation in the rat fed on indigestible oligosaccharides. Br J Nutr 80: 469-476.

41) Diehl GE, Longman RS, Zhang JX, Breart B, Galan C, Cuesta A, Schwab SR, Littman DR. 2013. Microbiota restricts trafficking of bacteria to mesenteric lymph nodes by CX(3) CR1(hi) cells. Nature 494(7435): 116-120.

42) Liu TW, Cephas KD, Holscher HD, Kerr KR, Mangian HF, Tappenden KA, Swanson KS. 2016. Nondigestible fructans alter gastrointestinal barrier function, gene expression, histomorphology, and the microbiota profiles of diet-induced obese C57BL/6J mice. J Nutr 146: 949-956. 